\title{
Delay-dependent dissipative control for stochastic singular systems with state delay
}

\author{
CHUNYAN DING and QIN LI
}

\begin{abstract}
The problem of delay-dependent dissipative analysis and control for stochastic singular systems with state delay is investigated in this paper. Delay-dependent dissipative condition for the stochastic singular systems with state delay is obtained by employing singular stochastic Lyapunov and LMI-based methods. Based on this condition, a delay-dependent dissipative controller is presented. A numerical example is provided to demonstrate the effectiveness of the proposed approach.
\end{abstract}

Key words: stochastic singular system, dissipative control, delay-dependent, state delay, linear matrix inequality (LMI)

\section{Introduction}

In the past decades, there have been considerable research efforts on the study of state delay systems since delays are often the main causes of instability and poor performance of dynamic systems and encountered in various engineering and physical systems. For more details regarding this class of systems, we refer the reader to [1,2] and the references therein. The existing results can be classified into two categories, delayindependent ones and delay-dependent ones. In particular, when the time-delay factor is known, it is emphasized that delay-dependent conditions yield less conservative performance results.

For the class of stochastic systems, a lot of works has been done[3,4,5,6]. Sufficient conditions on the stochastic stability and stochastic stabilizability were developed in [3]. The robust delay-dependent exponential stability of uncertain stochastic systems with time-varying delay was studied in [4]. On the other hand, for the class of singular systems, sufficient conditions on robust stability and the design of a state feedback controller have been derived by applying LMI technique in [7]. Stability problem for singular stochastic systems with Markovian switching has been considered in [8]. The problem for robust control of continues stochastic time-delay systems is discussed in [9].

C. Ding is with Yantai Engineering \& Technology College, Yantai, China. Q. Li is with School of Mathematics \& Information Science, Yantai University, Yantai, China. E-mails: dingchunyan@ gmail.com, qinli0412@163.com

Received 1.8.2013. 
To the best of our knowledge, there are few literature discussed the problem of dissipative control for stochastic singular systems with state delay.

In this work, we contribute to the development of dissipative analysis and synthesis of stochastic singular systems with state delay. First, a delay-dependent criterion will be presented to provide a sufficient condition for asymptotically stability in the mean-square sense and strict dissipativity. The desired state-feedback control law can be obtained by solving the feasibility problem of LMIs, which is very simple to do by using an interiorpoint algorithm[10].

Throughout this paper, the following notations will be used. The notation $\mathcal{E}\{\cdot\}$ for the expectation operator. " $*$ " is used as an ellipsis for terms that are induced by symmetry. $\|\cdot\|$ will refer to the Euclidean vector norm. Let $L_{2}[0, \infty)$ be the space of square integrable functions over $[0, \infty)$. While, $(\Omega, F, P)$ is a probability space, where $\Omega$ is the sample space, $F$ is the $\sigma$-algebra of subsets of the sample space and $P$ is the probability measure on $F$.

\section{Problem Formulation}

We consider a class of stochastic singular systems described by

$$
\begin{aligned}
(\Sigma): E d x(t) & =\left[A x(t)+A_{d} x(t-\tau)+B u(t)+A_{v} v(t)\right] d t+J x(t) d \omega(t) \\
y(t) & =C x(t) \\
z(t) & =L x(t)+D u(t)+D_{1} v(t) \\
x(t) & =\phi(t), \forall t \in[-\tau, 0]
\end{aligned}
$$

where $x(t) \in R^{n}$ is the state; $u(t) \in R^{m}$ is the control input; $y(t) \in R^{m}$ is the measured output; $z(t) \in R^{q}$ is the controlled output, $v(t) \in R^{p}$ is the disturbance input which belongs to $L_{2}[0, \infty) ; \omega(t)$ is a zero mean real scalar Wiener process on $(\Omega, F, P)$ relative to an increasing family $F_{t t>0}$ of $\sigma$-algebras $F_{t} \subset F$. We assume

$$
\mathcal{E}\{d \omega(t)\}=0 . \quad \mathcal{E}\left\{d \omega(t)^{2}\right\}=d t .
$$

$\tau>0$ represents constant time delay, $\phi(t)$ is a real-valued initial function on $[-\tau, 0]$. We denote by $L_{2}\left[\Omega, R^{k}\right)$ the space of square integrable $R^{k}$-valued vector function on the probability space $(\Omega, \mathcal{F}, \mathcal{P})$. We also denote by $L_{E_{2}}\left([0, \infty) ; R^{q}\right)$ the space of nonanticipatory square-integrable stochastic processes $f(\cdot)=(f(t))_{t \in[0, \infty)}$ in $R^{k}$ with respect to $\left(\mathcal{F}_{t}\right)_{t \in[0, \infty)}$ satisfying

$$
\|f\|_{E_{2}}^{2}=\mathcal{E}\left\{\int_{0}^{\infty}|f(t)| d t\right\}=\int_{0}^{\infty} \mathcal{E}\{|f(t)| d t\}<\infty .
$$

To facilitate the following discussion,we will introduce some definitions and lemmas, which are essential for the development of our main results. 
Definition 1 System $(\Sigma)$ with $u(t)=0$ and $v(t)=0$ is said to be mean-square stable if for any $\varepsilon>0$ there is $a \delta(\varepsilon)>0$ such that

$$
\mathcal{E}|x(t)|^{2}<\varepsilon, t>0
$$

when

$$
\sup _{-\mu \leqslant s \leqslant 0} \mathcal{E}|\phi(s)|^{2}<\delta(\varepsilon)
$$

If, in addition

$$
\lim _{t \rightarrow \infty} \mathcal{E}|x(t)|^{2}=0
$$

for any initial conditions, system $(\Sigma)$ with $u(t)=0$ and $v(t)=0$ is said to be mean-square asymptotically stable.

Definition 2 Given the supply rate $r(v(t), z(t))$, system $(\Sigma)$ of the form $(1)$ is said to be stochastic dissipative if there exists a $C^{0}$ nonnegative storage function $V(x(t), t)$, which satisfies $V(x(0))=0$, such that for all $v(t), z(t)$ and $t_{1} \geqslant t_{0}$

$$
\mathcal{E} \int_{t_{0}}^{t_{1}} \mathcal{L} V(x(t), t) d t \leqslant \mathcal{E} \int_{t_{0}}^{t_{1}} r(v(t), z(t)) d t .
$$

The above inequality is called the stochastic dissipation inequality. If the stochastic dissipation inequality holds strictly, then system $(\Sigma)$ is called to be stochastic strictly dissipative. In the note, we will consider quadratic supply rate

$$
r(v, z)=z^{T} Q z+2 z^{T} S v+v^{T} R v
$$

where $Q, S$ and $R$ are real matrices of appropriate dimensions with $Q$ and $R$ symmetric. Without the loss of generality, we set $Q=Q^{T}<0$.

Remark 3 It can be shown from definition 2 that the above strict dissipativity include $H_{\infty}$ performance and strict passivity as special cases.

1. When $Q=-I, S=0$ and $R=r^{2} I$, strict dissipativity reduces to $H_{\infty}$ performance requirement.

2. When $Q=0, S=I$ and $R=0$, strict dissipativity corresponds to strict passivity or strictly positive realness.

3. When $Q=-\theta I, S=(1-\theta) I, R=\theta r^{2} I, \theta \in(0,1)$, strict dissipativity represents mixed $H_{\infty}$ and positive real performance. 


\section{(a)}

C. DING, Q. LI

Lemma 5 [9] Consider the stochastic singular system described by

$$
E d x(t)=f(x(t), t) d t+g(x(t), t) d \omega(t)
$$

the function $f: R^{n} \times R^{+} \rightarrow R^{n}$ satisfy locally Lipschitz condition. Let $V(x(t), t)=$ $x^{T}(t) E^{T} P x(t)$, where $E^{T} P=P^{T} E \geqslant 0$, then the function $V(x(t), t)$ satisfies the following Itô formula:

$$
\begin{aligned}
d V(x(t), t) & =\left[f^{T}(x(t), t) P x(t)+x^{T}(t) P^{T} f(x(t), t)\right] d t \\
& +g^{T}(x(t), t)\left(E^{+}\right)^{T} E^{T} P E^{+} g(x(t), t) d t \\
& +2 x^{T}(t) P^{T} g(x(t), t) d \omega(t)
\end{aligned}
$$

where $E^{+}$stands for pseudo-inverse matrix.

Assumption $9 \operatorname{rank} E=\operatorname{rank}[E, J]$.

Assumption 10 The pair $(E, A)$ is regular and impulse free, i.e.

$$
\begin{aligned}
& \text { 1) } \operatorname{det}(s E-A) \neq 0, \\
& \text { 2) } \operatorname{deg}(\operatorname{det}-(s E-A))=\operatorname{rank} E .
\end{aligned}
$$

Lemma $6{ }^{[9]}$ Suppose Assumption 1 and Assumption 2 are satisfied, then the trivial solution to system $(\Sigma)$ exists and is continues and unique.

Lemma $7^{[11]}$ Given appropriate dimensional matrices $\widetilde{C}$ and $\widetilde{D}$ and a symmetric matrix $Z$, then

$$
Z+\widetilde{C} \Delta \widetilde{D}+\widetilde{D}^{T} \Delta^{T} \widetilde{C}^{T}<0
$$

for all $\Delta$ satisfying $\Delta^{T} \Delta \leqslant I$ if and only if there exists a scalar $\varepsilon>0$ such that

$$
Z+\varepsilon \widetilde{C} \widetilde{C}^{T}+\varepsilon^{-1} \widetilde{D}^{T} \widetilde{D}<0 .
$$

Lemma $8{ }^{[12]}$ For any symmetric positive definite matrix $R \in \mathbb{R}^{n \times n}$, a scalar $\tau>0$ and vector function $x(t):[0, \tau] \rightarrow \mathbb{R}^{n \times n}$, then

$$
-\tau \int_{t-\tau}^{t} x^{T}(s) R x(s) d s \leqslant-\int_{t-\tau}^{t} x^{T}(s) d s R \int_{t-\tau}^{t} x(s) d s .
$$




\section{Main Results}

In this section, a delay-dependent condition for system $(\Sigma)$ to be mean-square asymptotically stable and strictly stochastic dissipative is presented.

Theorem 11 System $(\Sigma)$ satisfies Assumptions 1,2. If there exist symmetric positive definite matrices $W, N$ and nonsingular matrix $P$, such that the following LMIs hold:

$$
\Theta=\left[\begin{array}{cccccc}
\Psi_{1} & P^{T} A_{d} & 0 & \Psi_{2} & L^{T} M^{T} & \tau A^{T} N \\
* & -W & 0 & 0 & 0 & \tau A_{d}^{T} N \\
* & * & -N & 0 & 0 & 0 \\
* & * & * & \Psi_{3} & 0 & \tau A_{v}^{T} N \\
* & * & * & * & -I & 0 \\
* & * & * & * & * & -N
\end{array}\right]
$$

where

$$
\begin{aligned}
& \Psi_{1}=A^{T} P+P^{T} A+W+J^{T}\left(E^{+}\right)^{T} E^{T} P E^{+} J \\
& \Psi_{2}=P^{T} A_{v}-L^{T} Q D_{1}-L^{T} S \\
& \Psi_{3}=-D_{1}^{T} Q D_{1}-D_{1}^{T} S-S^{T} D_{1}-R \\
& -Q=M^{T} M .
\end{aligned}
$$

Then it is mean-square asymptotically stable and strictly dissipative.

Proof The Assumption 1 and Assumption 2 guarantee that the trivial solution to system $(\Sigma)$ exists and is continues and unique in term of Lemma 2. Next we'll establish the mean-square asymptotical stability of system $(\Sigma)$ with $u(t)=0$ and $v(t)=0$. In this case, system $(\Sigma)$ becomes

$$
E d x(t)=\left[A x(t)+A_{d} x(t-\tau)\right] d t+J x(t) d \omega(t) .
$$

Set

$$
\begin{aligned}
& f_{1}(t)=A x(t)+A_{d} x(t-\tau) \\
& g_{1}(t)=A x(t)+A_{d} x(t-\tau)+A_{v} V(t) .
\end{aligned}
$$

Consider the following positive definite function as a Lyapunov functional candidate.

$$
V(t)=V_{1}(t)+V_{2}(t)+V_{3}(t)
$$


where

$$
\begin{aligned}
V_{1}(t) & =x^{T}(t) E^{T} P x(t) \\
V_{2}(t) & =\int_{t-\tau}^{t} x^{T}(\xi) W x(\xi) d \xi \\
V_{3}(t) & =\tau \int_{-\tau t+\eta}^{0} \int_{1}^{t} f_{1}^{T}(\xi) N f_{1}(\xi) d \xi d \eta \\
E^{T} P & =P^{T} E \geqslant 0, W>0, N>0 .
\end{aligned}
$$

Using the extended Itô formula according to Lemma 1, we obtain the stochastic differential as

$$
d V(x(t), t)=\mathcal{L} V d t+2 x^{T}(t) P^{T} J x(t) d \omega
$$

where

$$
\begin{aligned}
\mathcal{L} V(t) & =f_{1}^{T}(t) P x(t)+x^{T}(t) P^{T} f_{1}(t) \\
& +x^{T}(t) J^{T}\left(E^{+}\right)^{T} E^{T} P E^{+} J x(t)+x^{T}(t) W x(t) \\
& +x^{T}(t-\tau) W x(t-\tau)+\tau^{2} f_{1}^{T}(t) N f_{1}(t) \\
& -\tau \int_{t-\tau}^{t} f_{1}^{T}(\xi) N f_{1}(\xi) d \xi .
\end{aligned}
$$

Then by the Lemma 4, we can get

$$
\begin{aligned}
\mathcal{L} V(t) & \leqslant f_{1}^{T}(t) P x(t)+x^{T}(t) P^{T} f_{1}(t) \\
& +x^{T}(t) J^{T}\left(E^{+}\right)^{T} E^{T} P E^{+} J x(t)+x^{T}(t) W x(t) \\
& +x^{T}(t-\tau) W x(t-\tau)+\tau^{2} f_{1}^{T}(t) N f_{1}(t) \\
& -\tau \int_{t-\tau}^{t} f_{1}^{T}(\xi) d \xi N \int_{t-\tau}^{t} f_{1}(\xi) d \xi \\
& \leqslant \xi_{1}^{T}(t) \Theta_{1} \xi_{1}(t)
\end{aligned}
$$

where

$$
\xi_{1}^{T}(t)=\left[\begin{array}{lll}
x^{T}(t) & x^{T}(t-\tau) & \int_{t-\tau}^{t} f_{1}^{T}(\xi) d \xi
\end{array}\right]
$$

and

$$
\Theta_{1}=\left[\begin{array}{ccc}
\Psi_{1}+\tau^{2} A^{T} N A & P^{T} A_{d}+\tau^{2} A^{T} N A_{d} & 0 \\
* & -W+\tau^{2} A_{d}^{T} N A_{d} & 0 \\
* & * & -N
\end{array}\right]
$$


By the Schur complement, $\Theta<0$ is equivalent to

$$
\Theta_{2}=\left[\begin{array}{cccc}
\Psi_{1} & P^{T} A_{d} & 0 & \tau A^{T} N \\
* & -W & 0 & \tau A_{d}^{T} N \\
* & * & -N & 0 \\
* & * & * & -N
\end{array}\right]<0 .
$$

It is easy to obtain from (4) that $\Theta_{2}$ is negative-definite. From Definition 1 and [13], system $(\Sigma)$ is mean-square asymptotically stable.

Next, strictly stochastic dissipativity of $\operatorname{system}(\Sigma)$ with $u(t)=0$ will be discussed. The following positive definition function has been chosen as a Lyapunov function

$$
V(t)=V_{1}(t)+V_{2}(t)+\tau \int_{-\tau t+\eta}^{0} \int_{1}^{t} g_{1}^{T}(\xi) N g_{1}(\xi) d \xi d \eta .
$$

For any $v(t) \neq 0$, the stochastic differential will be obtained as

$$
d V(x(t), t)=\mathcal{L} V d t+2 x^{T}(t) P^{T} J x(t) d \omega
$$

where

$$
\begin{aligned}
\mathcal{L} V(t) & \leqslant g_{1}^{T}(t) P x(t)+x^{T}(t) P^{T} g_{1}(t) \\
& +x^{T}(t) J^{T}\left(E^{+}\right)^{T} E^{T} P E^{+} J x(t)+x^{T}(t) W x(t) \\
& -x^{T}(t-\tau) W x(t-\tau)+\tau^{2} g_{1}^{T}(t) N g_{1}(t) \\
& -\tau \int_{t-\tau}^{t} g_{1}^{T}(\xi) N g_{1}(\xi) d \xi
\end{aligned}
$$

Using Lemma 4,

$$
\begin{aligned}
\mathcal{L} V(t) & \leqslant g_{1}^{T}(t) P x(t)+x^{T}(t) P^{T} g_{1}(t) \\
& +x^{T}(t) J^{T}\left(E^{+}\right)^{T} E^{T} P E^{+} J x(t)+x^{T}(t) W x(t) \\
& -x^{T}(t-\tau) W x(t-\tau)+\tau^{2} g_{1}^{T}(t) N g_{1}(t) \\
& -\int_{t-\tau}^{t} g_{1}^{T}(\xi) d \xi N \int_{t-\tau}^{t} g_{1}(\xi) d \xi .
\end{aligned}
$$

For any $t>0$, introduce the cost function

$$
F(t)=\mathcal{E}\left\{\int_{0}^{t}\left[z^{T} Q z+2 z^{T} S v+v^{T} R v\right] d t\right\}
$$




$$
=\mathcal{E}\left\{\int_{0}^{t} \xi_{2}^{T}(t) \Pi_{2} \xi_{2}(t) d t\right\}
$$

where

$$
\begin{aligned}
& \xi_{2}^{T}(t)=\left[\begin{array}{llll}
x^{T}(t) & x^{T}(t-\tau) & \int_{t-\tau}^{t} g_{1}^{T}(\xi) d \xi & v^{T}(t)
\end{array}\right] \\
& \Pi_{2}=\left[\begin{array}{cccc}
L^{T} Q L & 0 & 0 & L^{T} Q D_{1}+L^{T} S \\
* & 0 & 0 & 0 \\
* & * & 0 & 0 \\
* & * & * & D_{1}^{T} Q D_{1}+D_{1}^{T} S+S^{T} D_{1}+R
\end{array}\right] .
\end{aligned}
$$

From Definition 2, we know that system $(\Sigma)$ is strictly dissipative if it holds

$$
\begin{gathered}
\mathcal{E}\left\{\int_{0}^{t} \mathcal{L} V(x(t), t) d t\right\}-F(t)<0 \\
\mathcal{E}\left\{\int_{0}^{t} \mathcal{L} V(x(t), t) d t\right\}-F(t) \\
=\mathcal{E}\left\{\int_{0}^{t} \mathcal{L} V(x(t), t) d t\right\}-\mathcal{E} \int_{0}^{t}\left[z^{T} Q z+2 z^{T} S v+v^{T} R v\right] d t \\
=\mathcal{E}\left\{\int_{0}^{t} \mathcal{L} V(x(t), t)-\left[z^{T} Q z+2 z^{T} S v+v^{T} R v\right] d t\right\} \\
\leqslant \mathcal{E}\left\{\int_{0}^{t} g_{1}^{T}(t) P x(t)+x^{T}(t) P^{T} g_{1}(t)+x^{T}(t) J^{T}\left(E^{+}\right)^{T} E^{T} P E^{+} J x(t)\right. \\
+x^{T}(t) W x(t)-x^{T}(t-\tau) W x(t-\tau)+\tau^{2} g_{1}^{T}(t) N g_{1}(t) \\
\left.-\int_{t}^{t} g_{1}^{T}(\xi) d \xi N \int_{t-\tau}^{t} g_{1}(\xi) d \xi-\left[z^{T} Q z+2 z^{T} S v+v^{T} R v\right] d t\right\}
\end{gathered}
$$




$$
\begin{aligned}
& =\mathcal{E}\left\{\int_{0}^{T} \xi_{2}^{T}(t) \Theta_{3} \xi_{2}(t)-F(t) d t\right\} \\
& =\mathcal{E}\left\{\int_{0}^{T} \xi_{2}^{T}(t) \Theta_{4} \xi_{2}(t) d t\right\}
\end{aligned}
$$

where

$$
\begin{aligned}
& \Theta_{3}=\left[\begin{array}{cccc}
\Psi_{4} & \Psi_{5} & 0 & P^{T} A_{v}+\tau^{2} A^{T} N A_{v} \\
* & \Psi_{6} & 0 & \tau^{2} A_{d}^{T} N A_{d} \\
* & * & -N & 0 \\
* & * & * & \tau^{2} A_{v}^{T} N A_{v}
\end{array}\right] \\
& \Theta_{4}=\left[\begin{array}{cccc}
\Psi_{4}-L^{T} Q L & \Psi_{5} & 0 & \Psi_{7} \\
* & \Psi_{6} & 0 & \tau^{2} A_{d}^{T} N A_{d} \\
* & * & -N & 0 \\
* & * & * & \Psi_{8}
\end{array}\right] \\
& \Psi_{4}=\Psi_{1}+\tau^{2} A^{T} N A \\
& \Psi_{5}=P^{T} A_{d}+\tau^{2} A^{T} N A_{d} \\
& \Psi_{6}=-W+\tau^{2} A_{d}^{T} N A_{d} \\
& \Psi_{7}=P^{T} A_{v}+\tau^{2} A^{T} N A_{v}-L^{T} Q D_{1}-L^{T} S \\
& \Psi_{8}=\tau^{2} A_{v}^{T} N A_{v}-D_{1}^{T} Q D-D_{1}^{T} S-S^{T} D_{1}-R \text {. }
\end{aligned}
$$

By the Schur complement, (4) is equivalent to (5). By using definition 2, it is obvious that system $(\Sigma)$ is strictly dissipative. This completes the proof.

We are now in a position to present the result of stochastic dissipative stabilization. The objective is to develop an LMI-based solution to the problem of designing a state-feedback controller $u(t)=K x(t)$ which will render system $(\Sigma)$ strictly stochastic dissipative. The closed-loop system is now described by

$$
\begin{aligned}
\left(\Sigma_{c}\right): E d x(t) & =\left[(A+B K) x(t)+A_{d} x(t-\tau)+A_{v} v(t)\right] d t+J x(t) d \omega(t) \\
z(t) & =(L+D K) x(t)+D_{1} v(t) .
\end{aligned}
$$

Theorem 12 Consider system $(\Sigma)$ subject to Assumptions 1,2. If there exist a scalar $\varepsilon>$ 0 , symmetric positive definite matrices $W, N$, nonsingular matrix $P$ and $\widetilde{K}>0$ satisfying the following LMIs:

$$
E^{T} P=P^{T} E \geqslant 0
$$




$$
\left[\begin{array}{ccccccc}
\Psi_{1}+\widetilde{K} & P^{T} A_{d} & 0 & \Psi_{2} & L^{T} M^{T} & \tau A^{T} N & P^{T} B \\
* & -W & 0 & 0 & 0 & \tau A_{d}^{T} N & 0 \\
* & * & -N & 0 & 0 & 0 & 0 \\
* & * & * & \Psi_{3} & 0 & \tau A_{v}^{T} N & \Psi_{9} \\
* & * & * & * & -I & 0 & M D \\
* & * & * & * & * & -N & \tau N^{T} B \\
* & * & * & * & * & * & -\varepsilon I
\end{array}\right]<0
$$

where

$$
\Psi_{9}=-D_{1}^{T} Q D-S^{T} D .
$$

Then there exists state feedback controller for system $(\Sigma)$ such that the resulting closedloop system is mean-square asymptotically stable and strictly dissipative. And the controller gain is the symmetrical partition of the matrix $\widetilde{K} / \varepsilon$.

Proof Applying the state feedback controller $u(t)=K x(t)$, system $(\Sigma)$ can be written as $\left(\Sigma_{c}\right)$. By Theorem 1 , system $\left(\Sigma_{c}\right)$ is mean-square asymptotically stable and strictly dissipative if there exists matrices $P, W>0$ and $N>0$ satisfying (3) and

$$
\left[\begin{array}{cccccc}
\Psi_{10} & P^{T} A_{d} & 0 & \Psi_{11} & (L+D K)^{T} M^{T} & \tau(A+B K)^{T} N \\
* & -W & 0 & 0 & 0 & \tau A_{d}^{T} N \\
* & * & -N & 0 & 0 & 0 \\
* & * & * & \Psi_{3} & 0 & \tau A_{v}^{T} N \\
* & * & * & * & -I & 0 \\
* & * & * & * & * & -N
\end{array}\right]<0
$$

where

$$
\begin{aligned}
& \Psi_{10}=A^{T} P+P^{T} A+K^{T} B^{T} P+P^{T} B K+W+J^{T}\left(E^{+}\right)^{T} E^{T} P E^{+} J \\
& \Psi_{11}=P^{T} A_{v}-(L+D K)^{T} Q D_{1}-(L+D K)^{T} S .
\end{aligned}
$$

The inequality (8) can be written as

$$
\Theta+\Xi^{T} \Gamma+\Gamma^{T} \Xi<0
$$

where

$$
\begin{gathered}
\Xi=\left[\begin{array}{llllll}
K & 0 & 0 & 0 & 0 & 0
\end{array}\right] \\
\Gamma=\left[\begin{array}{llllll}
B^{T} P & 0 & 0 & -D^{T} Q D_{1}-D^{T} S & D^{T} M^{T} & \tau B^{T} N
\end{array}\right] .
\end{gathered}
$$


Now, applying Lemma 3, we have

$$
\Theta+\varepsilon \Xi^{T} \Xi+\varepsilon^{-1} \Gamma^{T} \Gamma<0 .
$$

Then, using Schur complement, it results that inequality (10) is equivalent to inequality (7). The proof is completed.

\section{Numerical example}

Consider a second-order system of the type $(\Sigma)$, where the nominal model matrices are

$$
\begin{gathered}
E=\left[\begin{array}{ll}
1 & 0 \\
0 & 0
\end{array}\right], A=\left[\begin{array}{cc}
-20 & -5 \\
8 & -15
\end{array}\right], A_{v}=\left[\begin{array}{cc}
0.8 & 2 \\
1 & -2
\end{array}\right], \\
A_{d}=\left[\begin{array}{cc}
0.4 & 1 \\
0 & 0.5
\end{array}\right], B=\left[\begin{array}{cc}
2 & -4.2 \\
0 & 1
\end{array}\right], J=\left[\begin{array}{cc}
10 & 1 \\
2 & -1
\end{array}\right], \\
L=\left[\begin{array}{cc}
3 & -2 \\
6 & 9
\end{array}\right], D=\left[\begin{array}{cc}
15 & 0 \\
3 & 3
\end{array}\right], D_{1}=\left[\begin{array}{cc}
1 & 10 \\
0 & 0.5
\end{array}\right] .
\end{gathered}
$$

The parameters of supply rate are given:

$$
Q=\left[\begin{array}{cc}
-1 & 0 \\
0 & -4
\end{array}\right], S=\left[\begin{array}{cc}
1 & 9 \\
2 & 1.5
\end{array}\right], R=\left[\begin{array}{ll}
4 & 1 \\
1 & 3
\end{array}\right] .
$$

Using the LMI-solver, a feasible solution of Theorem 2 shows that the system is delay-dependent mean-square asymptotically stable and strictly stochastic dissipative for $\tau<0.206$. When $\tau=0.1$, the solutions of LMIs (6), (7) are as following:

$$
\begin{gathered}
P=\left[\begin{array}{cc}
85.1213 & 0 \\
-16.7537 & 45.8078
\end{array}\right], W=\left[\begin{array}{cc}
989.1832 & -160.1246 \\
-160.1246 & 289.2770
\end{array}\right], \\
N=\left[\begin{array}{cc}
302.3339 & -16.6159 \\
-16.6159 & 158.5975
\end{array}\right], \widetilde{K}=\left[\begin{array}{cc}
584.6290 & -100.2020 \\
-100.2020 & 147.9065
\end{array}\right] .
\end{gathered}
$$

And the controller gain is

$$
K=\left[\begin{array}{cc}
0.4110 & -0.0704 \\
0 & 0.1943
\end{array}\right]
$$




\section{Conclusions}

In this paper, the problem of delay-dependent dissipative analysis and control for stochastic singular systems with state delay has been studied. By the extended It $\hat{o}$ stochastic differential formula method, a delay-dependent strictly stochastic dissipative condition has been obtained, and a delay-dependent controller has been presented. The results were presented in terms of LMIs, which can be solved easily by using the effective interior-point algorithm. A numerical example demonstrated that the theoretical results are correct and effective.

\section{References}

[1] M.S. Mahmoud: Delay-dependent dissipativity of singular time-delay systems. IMA J. of Mathematical Control and Information, 26(1), (2009), 45-58.

[2] C. LI and X. LIAO: Passivity analysis of neural networks with time delay. IEEE Trans. on Circuits and Systems, 52(8), (2005), 471-475.

[3] E.K. Boukas, S. XU and J. LAM: On stability and stabilizability of singular stochastic systems with delays. J. of Optimization Theory and Applications, 127(2), (2005), 249-262.

[4] M. Hua, F. Deng and Y. Peng: Robust delay-dependent exponential stability of uncertain stochastic system with time-varying delay. Circuits Systems and Signal Process, 29(3), (2010), 515-526.

[5] Z.J. WU, M.Y. CUI, X.J. XIE and P. SHI: Theory of stochastic dissipative systems. IEEE Trans. on Automatic Control, 56(7), (2011), 1650-1655.

[6] W. LI, X. XIE and S. ZHANG: Output-feedback stabilization of stochastic highorder nonlinear systems under weaker conditions. SIAM J. on Control and Optimization, 49(3), (2011), 1262-1282.

[7] J. RAOUF and E. K. BoukAS: Roubst stabilization of Markovian jump linear singualr systems with Wiener process. In Proc. of the 2004 American Control Conf., 4 (2004), 3170-3175.

[8] L. HUANG and X. MAO: Stability of singular stochastic systems with Markovian switching. IEEE Trans. on Automatic Control, 56(2), (2011), 424-429.

[9] J.W. XIA: The robust $H_{\infty}$ filter design for uncertain time-delay singular stochastic systems with Markovian jump. J. of Control Theory and Applications, 5(1), (2007), 331-335. 
[10] S. Boyd, L. E. et al.; Linear Matrix Inequalities in Syetem and Control Theory, Philadelphia, PA: SIAM, 1994.

[11] L. XIE, M.Y. FU and C.E. DE SouZA: $H_{\infty}$ control and quadratic stabilization of systems with parameter uncertainty via output feedback. IEEE Trans. on Automatic Control, 37(8), (1992), 1253-1256.

[12] K.Q. Gu, V.L. Kharitonov and J. Chen: Stability of Time-delay Systems. Birkhäuser, Boston, 2003.

[13] V.B. Kolmanovski and A.D. Myshkis: Applied Theory of Functional Differential Equations. Dordreclu, The Netherlands, Kluwer, 1992. 